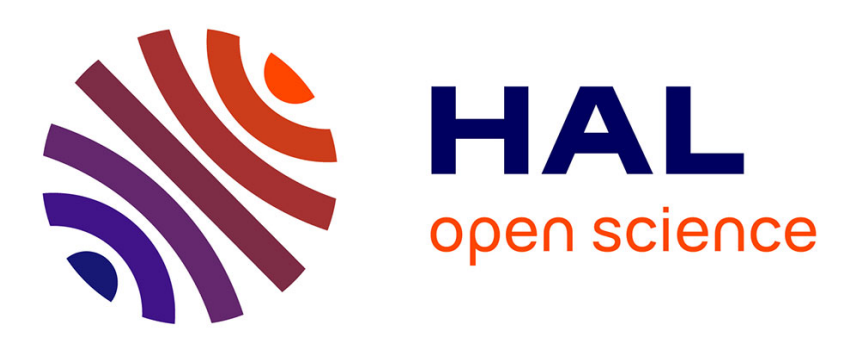

\title{
Magnetization switching diagram of a perpendicular synthetic ferrimagnet $\mathrm{CoFeB} / \mathrm{Ta} / \mathrm{CoFeB}$ bilayer
}

O Koplak, A Talantsev, Yuan Lu, Abbass Hamadeh, Philipp Pirro, Thomas

Hauet, R Morgunov, Stéphane Mangin

\section{- To cite this version:}

O Koplak, A Talantsev, Yuan Lu, Abbass Hamadeh, Philipp Pirro, et al.. Magnetization switching diagram of a perpendicular synthetic ferrimagnet $\mathrm{CoFeB} / \mathrm{Ta} / \mathrm{CoFeB}$ bilayer. Journal of Magnetism and Magnetic Materials, 2017, 433, pp.91-97. 10.1016/j.jmmm.2017.02.047 . hal-01569342

\section{HAL Id: hal-01569342 \\ https://hal.science/hal-01569342}

Submitted on 26 Jul 2017

HAL is a multi-disciplinary open access archive for the deposit and dissemination of scientific research documents, whether they are published or not. The documents may come from teaching and research institutions in France or abroad, or from public or private research centers.
L'archive ouverte pluridisciplinaire HAL, est destinée au dépôt et à la diffusion de documents scientifiques de niveau recherche, publiés ou non, émanant des établissements d'enseignement et de recherche français ou étrangers, des laboratoires publics ou privés. 


\section{Manuscript Details}

\section{Manuscript number}

Title

\author{
MAGMA_2017_228
}

Magnetization switching diagram of a perpendicular synthetic ferrimagnet CoFeB/Ta/CoFeB bilayer

Full Length Article

\section{Article type}

Abstract

Magnetic configurations in synthetic ferrimagnet $\mathrm{CoFeB} / \mathrm{Ta} / \mathrm{CoFeB}$ bilayer with strong perpendicular anisotropy have been systematically studied. Magnetization versus field hysteresis loop has been measured for different temperature ranging from 5 to $300 \mathrm{~K}$. The applied field - temperature $(\mathrm{H}-\mathrm{T})$ magnetization switching diagram has been constructed by extracting the different switching fields as a function of temperature. This switching diagram can be well explained by considering the competition between energy barrier of layer's magnetization reversal, interlayer exchange coupling, and Zeeman energy.

\section{Keywords}

\section{Corresponding Author \\ Corresponding Author's Institution}

Order of Authors

Suggested reviewers synthetic antiferromagnetic; perpendicular magnetic anisotropy; magnetic switching diagram

\author{
Yuan LU \\ Universite de Lorraine
}

Yuan LU, Oksana Koplak, Artem Talantsev, Abbass HAMADEH, Philipp Pirro, Thomas Hauet, Roman Morgunov, Stephane Mangin

Gerd Schoenhense, Hiroko Tokoro, Michael Farle, Eric Fullerton, Alberta Bonanni

\section{Submission Files Included in this PDF}

File Name [File Type]

Cover letter-revision_JMMM.doc [Cover Letter]

Answer to referee comment.docx [Response to Reviewers]

JMMM_Mapping1_improved.docx [Manuscript File]

fig1.JPG [Figure]

fig2.jpg [Figure]

fig3.jpg [Figure]

fig4.JPG [Figure]

fig5.jpg [Figure]

fig6.JPG [Figure]

fig7.JPG [Figure]

Highlights.doc [Highlights]

To view all the submission files, including those not included in the PDF, click on the manuscript title on your EVISE Homepage, then click 'Download zip file'. 


\section{Cover Letter}

Professor Roman Morgunov,

Institute of Problems of Chemical Physics

of the Russian Academy of Sciences,

1, Academician Semenov's avenue, Chernogolovka,

142432, Moscow region, Russia,

morgunov2005@yandex.ru

Editor, Journal of Magnetism and Magnetic materials

Feb. 21, 2017

Dear Editor,

We are writing to resubmit our revised manuscript entitled "Magnetization state diagram in a perpendicular synthetic ferrimagnet $\mathrm{CoFeB} / \mathrm{Ta} / \mathrm{CoFeB}$ trilayer" for consideration by the Journal of Magnetism and Magnetic materials.

We have answered the question of referee in the response letter and make corresponding change in the manuscript.

Thank you for your consideration!

Best regards,

Authors 
Authors are thankful to referee for his comment.

To clarify how bottom and upper layers were distinguished, we introduced following sentence to text (page 3).

To identify the switching sequence for bottom and top CoFeB layers from $\mathrm{M}-\mathrm{H}$ curves, we have performed spin-LED measurement, which is only sensitive to the magnetization state of the bottom CoFeB layer. In addition, the total manetic dead layer is determined to be about $0.5 \mathrm{~nm}$ which is almost equally distributed in both sides of Ta insertion layer [B. S. Tao, P. Barate, J. Frougier, P. Renucci, B. Xu, A. Dje_al, H. Jaffès, J.-M. George, X. Marie, S. Petit-Watelot, S. Mangin, X. F. Han, Z. G. Wang, and Y. Lu, Appl. Phys. Lett. 108, 152404 (2016)]. 


\title{
Magnetization switching diagram of a perpendicular synthetic ferrimagnet $\mathrm{CoFeB} / \mathrm{Ta} / \mathrm{CoFeB}$ bilayer
}

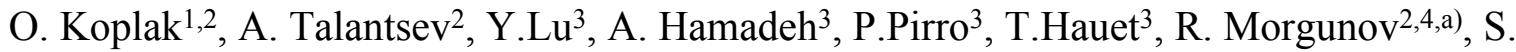 \\ Mangin $^{3}$ \\ ${ }^{1}$ Immanuel Kant Baltic Federal University, 236041, Kaliningrad, Russia \\ ${ }^{2}$ Institute of Problems of Chemical Physics, 142432, Chernogolovka, Moscow, Russia \\ ${ }^{3}$ Institut Jean Lamour, UMR 7198 CNRS, Université de Lorraine, France \\ ${ }^{4}$ Tambov State Technical University, 392000, Tambov, Russia
}

Magnetic configurations in synthetic ferrimagnet $\mathrm{CoFeB} / \mathrm{Ta} / \mathrm{CoFeB}$ bilayer with strong perpendicular anisotropy have been systematically studied. Magnetization versus field hysteresis loop has been measured for different temperature ranging from 5 to $300 \mathrm{~K}$. The applied field - temperature (H-T) magnetization switching diagram has been constructed by extracting the different switching fields as a function of temperature. This switching diagram can be well explained by considering the competition between energy barrier of layer's magnetization reversal, interlayer exchange coupling, and Zeeman energy.

\section{INTRODUCTION}

Ferromagnetic layers exchange coupled antiferromagnetically known as Synthetic antiferromagnets (SAF) are currently used in technologically relevant systems such as magnetic random access memories (MRAMs) and read heads for magnetic data storage $\left.{ }^{1}\right]$. SAF using materials with perpendicular magnetic anisotropy (PMA) are now used to combine higher densities, thermal stability and sharp magnetic switching. It is also expected to be used to reduce the critical current density in Spin-Transfer-Torque (STT) MRAM. Since the prediction of large domain wall velocity [ $\left.{ }^{2}\right]$ and the possibility of all Optical switching PMA-SAF $\left[{ }^{3-4}\right]$ are attracting a lot of attention. Multilayer devices based on CoFeB ferromagnetic layers separated by a thin nonmagnetic layer attract attention of specialists due to various possible fields of practical application. Metallic spintronics based on CoFeB multilayered structures rapidly gains new areas of its application in sensor industry $\left.{ }^{5-6}\right]$, biomedicine, and information technologies $\left[{ }^{7-8}\right]$. Magnetic structure of $\mathrm{CoFeB}$ single layers

\footnotetext{
a Correspondent author e-mail: morgunov2005@yandex.ru
} 
have been studied in detail $\left[{ }^{9-14}\right]$. Numerous reports on methods for inducing domain walls by electric current $\left[{ }^{15-16}\right]$, manipulating them $\left[{ }^{17-19}\right]$, and reversing polarity of perpendicularly polarized nanosized single- and multilayer magnets without external magnetic field $\left[{ }^{20}\right]$ by means of local electric fields $\left[{ }^{21-22}\right]$ and currents $\left[{ }^{23-25}\right]$ can be found for single $\mathrm{CoFeB}$ magnets. However, magnetization reversal of multilayer magnetic structures remains a challenge due to a complicated game of anisotropy of layers $\left[{ }^{26-30}\right]$, DMI interaction $\left[{ }^{31-32}\right]$, tensile strains $\left[{ }^{33-34}\right]$ and interlayer coupling $\left.{ }^{35-36}\right]$. The magnetic configurations present in in-plane antiferromagnetically exchange coupled bilayers, as well as bilayers with PMA, have been studied in the past $\left.{ }^{37-39}\right]$. In the recent papers $\left[{ }^{35-36}\right]$ dependence of the exchange interaction value and sign on the thickness of non magnetic spacer was demonstrated. However, one of the unclear questions is determination of correspondence between the energy balance of key interactions (exchange coupling $E_{\mathrm{EX}}$, Zeeman energy $E_{\mathrm{Z}}$ and barriers of reversal magnetization $E_{\text {eff1 }}$ and $E_{\text {eff2}}$ ) and sequence of the magnetization switching under external magnetic field. Here we will demonstrate that sequence of the magnetization switching depends on the ratio between the exchange coupling and barrier of reversal magnetization.

Convenient diagram technique to predict sequence of switching of the bilayered devices depending on the ratio of layers magnetization and potential barriers of the reversal magnetization is proposed in our article. Sequence of the magnetization switching and shape of the hysteresis loop as well as prohibited and permitted transitions and their threshold magnetic fields controlled by competition between $E_{\mathrm{EX}}, E_{\mathrm{Z}}, E_{\mathrm{eff1}}$ and $E_{\mathrm{eff2}}$ values has been determined in our work. The experimental results are summarized in switching diagram of the magnetization states and transitions between different states depending on temperature $T$, applied field $H$ and the thermal and magnetic prehistory in a perpendicularly magnetized antiferromagnetically exchange coupled $\mathrm{CoFeB} / \mathrm{Ta} / \mathrm{CoFeB}$ bilayer.

\section{EXPERIMENTAL}

The multilayer system which consists of $\mathrm{MgO}(2.5) / \mathrm{CoFeB}(1.1) / \mathrm{Ta}(0.75) / \mathrm{CoFeB}(0.8) / \mathrm{MgO}(2.5) / \mathrm{Ta}(5)$ (unit of nm) was deposited on the undoped GaAs (001) substrate (Figure 1) by magnetron sputtering. The GaAs substrate was selected for easy integration into semiconductor based devices, such as spin LED structures $\left[{ }^{40-41}\right]$, and for future semiconductor spintronics application. Growth conditions are 
described in detail in [ $\left.{ }^{42-43}\right]$. In the investigated layer stack, the hybridization of the $3 \mathrm{~d}$ orbitals of transition metals $(\mathrm{Co}, \mathrm{Fe})$ with the $\mathrm{O}_{2 p}$ orbitals of $\mathrm{MgO}$ provides perpendicular magnetic anisotropy (PMA) at the $\mathrm{CoFeB} / \mathrm{MgO}$ interface $\left[{ }^{44}\right]$. To enhance PMA, rapid temperature annealing (RTA) was performed at $250^{\circ} \mathrm{C}$ for 3 min to crystallize the $\mathrm{CoFeB}$ layer. During this process, the diffusing boron was absorbed by the $0.75 \mathrm{~nm}$ Ta interlayer, which also provided antiferromagnetic coupling between the two adjacent CoFeB layers $\left[{ }^{42}\right.$, ${ }^{45}$ ]. The plate-shaped sample $0.4 \times 4 \times 4 \mathrm{~mm}^{3}$ in size was studied in experiments. Magnetic hysteresis loops were obtained with an MPMS 5XL Quantum Design superconducting quantum interference device (SQUID) magnetometer.

\section{RESULTS AND DISCUSSION}

The magnetic hysteresis loops of the bilayer system at $T=300 \mathrm{~K}, 150 \mathrm{~K}$ and $50 \mathrm{~K}$ are presented in the Figure 2. The insert in the Fig. 2a summarizes four different states (two parallel $\uparrow \uparrow, \downarrow \downarrow$ and two antiparallel $\uparrow \downarrow, \downarrow \uparrow$ states) corresponding to different magnetization orientations in agreement with our previous study $\left[{ }^{40}\right]$. To identify the switching sequence for bottom and top CoFeB layers from M-H curves, we have performed spin-LED measurement, which is only sensitive to the magnetization state of the bottom CoFeB layer. In addition, the total magnetic dead layer is determined to be about $0.5 \mathrm{~nm}$ which is almost equally distributed in both sides of Ta insertion layer[ $\left.{ }^{40}\right]$. The left arrow corresponds to the bottom $(1.1 \mathrm{~nm})$ thick layer magnetization. The right arrow corresponds to the top $(0.8 \mathrm{~nm})$ thin layer magnetization. The upright direction of the arrow corresponds to magnetization along the positive direction of magnetic field. Magnetic moments of the bilayer corresponding to four magnetic states are:

$$
M_{\uparrow \uparrow}=M_{1}+M_{2} ; M_{\uparrow \downarrow}=M_{1}-M_{2} ; M_{\downarrow \uparrow}=-M_{1}+M_{2} ; M_{\downarrow \downarrow}=-M_{1}-M_{2} .
$$

where $\boldsymbol{M}_{\mathbf{1}}$ and $\boldsymbol{M}_{\mathbf{2}}$ are saturated magnetic moments of the bottom and top layers, respectively. The condition $\left|M_{2}\right|<\left|M_{1}\right|$ results in positive $\boldsymbol{M}_{\uparrow \uparrow}$ and $\boldsymbol{M}_{\uparrow \downarrow}$ values and negative $\boldsymbol{M}_{\downarrow \uparrow}$ and $\boldsymbol{M}_{\downarrow \downarrow}$ values in our experiments (fig.2). 


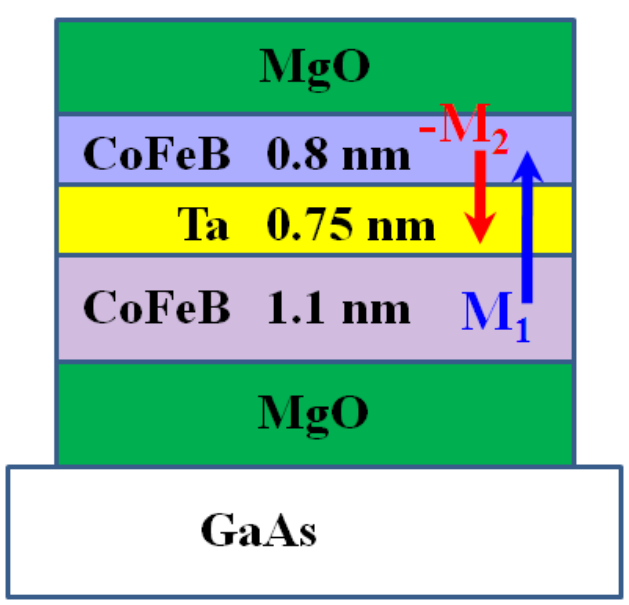

Figure 1. The sketch of the $\mathrm{CoFeB} / \mathrm{Ta} / \mathrm{CoFeB}$ bilayer deposited on the $\mathrm{GaAs}$ substrate. $\mathrm{MgO}$ - cap and buffer layers. $\mathrm{CoFeB}$ layers are coupled antiferromagnetically. $\boldsymbol{M}_{\mathbf{1}}$ and $\boldsymbol{M}_{\mathbf{2}}$ arrows correspond to bottom and top layers' magnetizations, respectively.
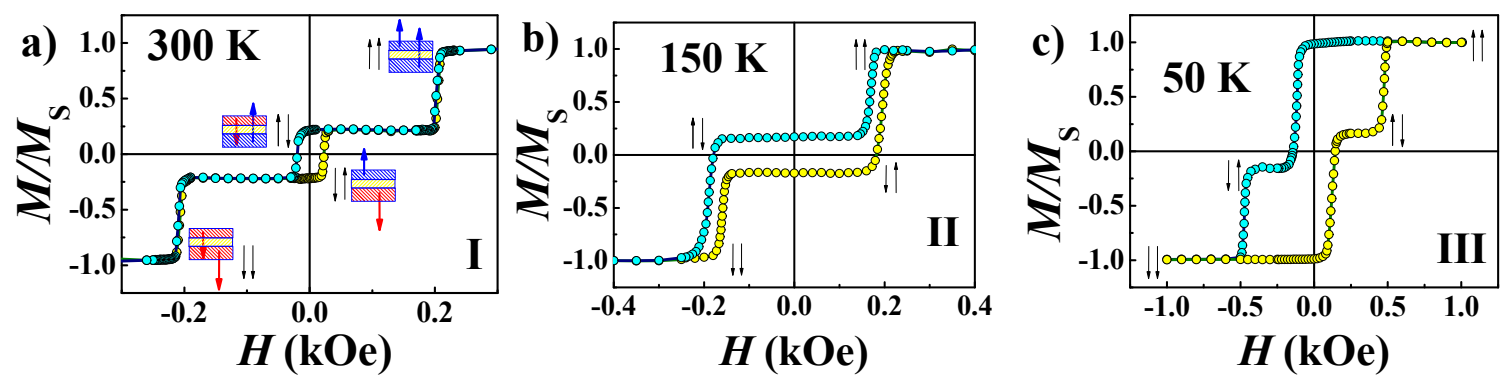

Figure 2. Magnetic hysteresis loops at $300 \mathrm{~K}$ (a), $150 \mathrm{~K}$ (b), and $50 \mathrm{~K}$ (c). The arrows indicate layers' magnetization directions at the stable magnetic states of bilayer. The symbols of the blue color correspond to top-down magnetic field sweeping direction and yellow symbols - to the bottom up field sweeping direction.

It is interesting to see the hysteresis loops are completely different depending on the temperature ranges. The complicate shape of the hysteresis loop recorded at $300 \mathrm{~K}$ is due to successive switching between aforementioned magnetic states $(\uparrow \uparrow, \uparrow \downarrow, \downarrow \uparrow, \downarrow \downarrow)$. Three correspondent transitions can be distinguished in the Figure 2:

- single reversal of top layer from ferromagnetic state $\uparrow \uparrow$ to antiferromagnetic one $\uparrow \downarrow$ at +200 Oe;

- double simultaneous reversal of both, top and bottom layers from antiferromagnetic state $\uparrow \downarrow$ at -20 Oe;

- single reversal of top layer from antiferromagnetic state $\downarrow \uparrow$ to ferromagnetic one $\downarrow \downarrow$ at -210 Oe. Bottom layer single reversal does not occur independently on magnetic field sweeping interval and direction.

At $150 \mathrm{~K}$ the hysteresis loop contains two transitions: single reversal of top layer (from $\uparrow \uparrow$ to $\uparrow \downarrow$ ) at +200 Oe and single reversal of the bottom layer (from $\uparrow \downarrow$ to $\downarrow \downarrow$ ) at -250 Oe. 
Additional transition is possible, if the magnetic field sweeping direction is reversed before then transition from $\uparrow \downarrow$ to $\downarrow \downarrow$ occurs (see minor hysteresis loops, Figure 3 ). In that case top layer single reversal from antiferromagnetically coupled state $(\uparrow \downarrow \Rightarrow \uparrow \uparrow)$ occurs. However, simultaneous reversals of top and bottom layers, as well as bottom layer single reversal from ferromagnetically coupled state (from $\uparrow \uparrow$ to $\downarrow \uparrow$ ) do not occur, independently on the field sweeping range and direction.
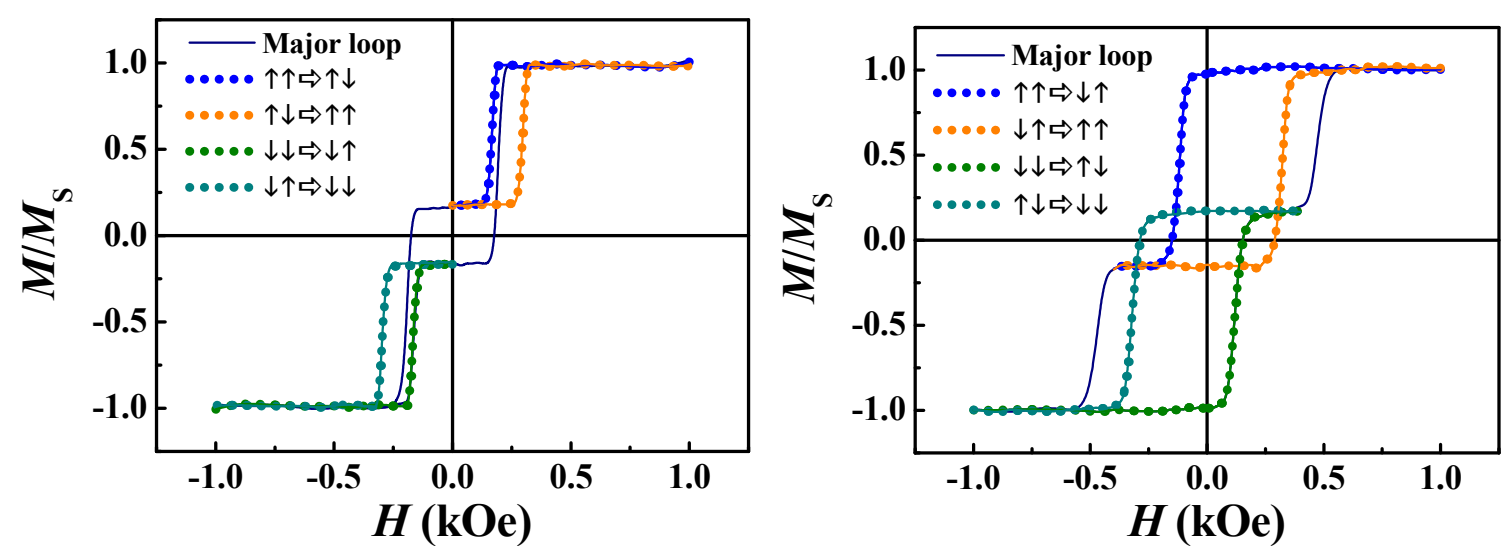

Figure 3. Minor magnetic hysteresis loops at $150 \mathrm{~K}$ (a) and $50 \mathrm{~K}$ (b). Right loop corresponds to $H_{\mathrm{S}} \Rightarrow H_{0} \Rightarrow H_{\mathrm{S}}$ field sweeping direction, left loop corresponds to $-H_{\mathrm{S}} \Rightarrow-H_{0} \Rightarrow-H_{\mathrm{S}}, H_{\mathrm{S}}-$ saturation field, $H_{0}$ - arbitrary field between the first and second transition on the top-down branch of the major magnetic hysteresis loop (shown as solid background line).

The hysteresis loop contains two other transitions at $50 \mathrm{~K}$ : single reversal of the bottom layer (from $\uparrow \uparrow$ to $\downarrow \uparrow$ ) at -100 Oe and single reversal of top layer (from $\downarrow \uparrow$ to $\downarrow \downarrow$ ) at -450 Oe. Additional transition from $\uparrow \downarrow$ to $\uparrow \uparrow$ occurs at +340 Oe if magnetic sweep direction was changed to opposite one (see minor hysteresis loops in the Figure 3). However, simultaneous reversals of top and bottom layers, as well as single reversal of the top layer from $\uparrow \uparrow$ to $\uparrow \downarrow$ do not occur, independently on the field sweeping range and direction. 


\begin{tabular}{|c|c|c|c|c|}
\hline \multirow{2}{*}{ Transitions } & \multirow{2}{*}{ Description } & \multicolumn{3}{|c|}{ Temperature range $(\mathrm{K})$} \\
\hline & & $2-110$ & $120-170$ & $180-330$ \\
\hline $\begin{array}{l}\uparrow \uparrow \Rightarrow \uparrow \downarrow \\
\downarrow \downarrow \Rightarrow \downarrow \uparrow\end{array}$ & $\begin{array}{l}\text { Top layer single reversal } \\
\text { from parallel state }\end{array}$ & & & \\
\hline $\begin{array}{l}\uparrow \downarrow \Rightarrow \uparrow \uparrow \\
\downarrow \uparrow \Rightarrow \downarrow \downarrow\end{array}$ & $\begin{array}{l}\text { Top layer single reversal } \\
\text { from antiparallel state }\end{array}$ & & & \\
\hline $\begin{array}{l}\uparrow \uparrow \Rightarrow \downarrow \uparrow \\
\downarrow \downarrow \Rightarrow \uparrow \downarrow\end{array}$ & $\begin{array}{c}\text { Bottom layer single } \\
\text { reversal } \\
\text { from parallel state }\end{array}$ & & & \\
\hline $\begin{array}{l}\uparrow \downarrow \Rightarrow \uparrow \uparrow \\
\downarrow \uparrow \Rightarrow \downarrow \downarrow\end{array}$ & $\begin{array}{c}\text { Bottom layer single } \\
\text { reversal } \\
\text { from antiparallel state }\end{array}$ & & & \\
\hline $\begin{array}{l}\uparrow \downarrow \Rightarrow \downarrow \uparrow \\
\downarrow \uparrow \Rightarrow \uparrow \downarrow\end{array}$ & $\begin{array}{l}\text { Top and bottom layer } \\
\text { simultaneous reversal } \\
\text { from antiparallel state }\end{array}$ & & & \\
\hline $\begin{array}{l}\uparrow \uparrow \Rightarrow \downarrow \downarrow \\
\downarrow \downarrow \Rightarrow \uparrow \uparrow\end{array}$ & $\begin{array}{l}\text { Top and bottom layer } \\
\text { simultaneous reversal } \\
\text { from parallel state }\end{array}$ & & & \\
\hline
\end{tabular}

Table 1. Temperature map of magnetic transitions. White squares correspond to forbidden transitions at the given temperature range. Green squares means that transition is permitted and occurs at the definite magnetic field independently on the magnetic field sweeping range. Yellow squares correspond to permitted transitions, which occur only in the minor hysteresis loop.

Thus, totally one can discuss 12 transitions between 4 different magnetization states. Just six non equivalent transitions of the twelve exist due to symmetry with respect to the direction of magnetic field sweep. Direct transition between $\uparrow \uparrow$ and $\downarrow \downarrow$ states (simultaneous reversal from ferromagnetic state) does not occur in the entire $2-300 \mathrm{~K}$ temperature range. The rest five transitions do not appear at any temperature simultaneously. Just three of them can be observed at any temperature. Series of the permitted and forbidden transitions for each temperature interval $(2-110 \mathrm{~K}, 120-170 \mathrm{~K}$ and $180-330 \mathrm{~K})$ are summarized in Table 1. Temperature dependences of the threshold magnetic fields for all 12 transitions are presented in the Figure $3 b$.

Threshold magnetic fields were extracted from the hysteresis loops. Every point in the Figure $3 \mathrm{~b}$ correspond temperature at which loop was recorded and threshold field of sharp change of magnetization. Additionally we checked another way to obtain points for the Figure $3 \mathrm{~b}$. Same values of the transition temperature and magnetic field were extracted from temperature dependence of magnetization recorded during heating of the sample which was preliminary cooled down to $2 \mathrm{~K}$ in the different magnetic fields. Examples of these temperature dependences are presented in Supporting materials (Figure S1). Magnetic field 
applied during temperature dependence recording was stable. Switching of the states was identified by sharp change of magnetization when temperature reached threshold value.

Switching of the magnetic states was observed on the temperature dependence during sample heating only (see Figures S1 and S2 of the Supporting materials). Decrease of the temperature retains initial magnetic state existing in the sample before its cooling. One can consider cooling of the bilayer device as a method of storage of the magnetic information recorded at high temperature. Numeration 1-4 in the Figure $3 \mathrm{~b}$ corresponds to number of the magnetic states, which can be stored under cooling in the determined magnetic field down to the indicated temperature.

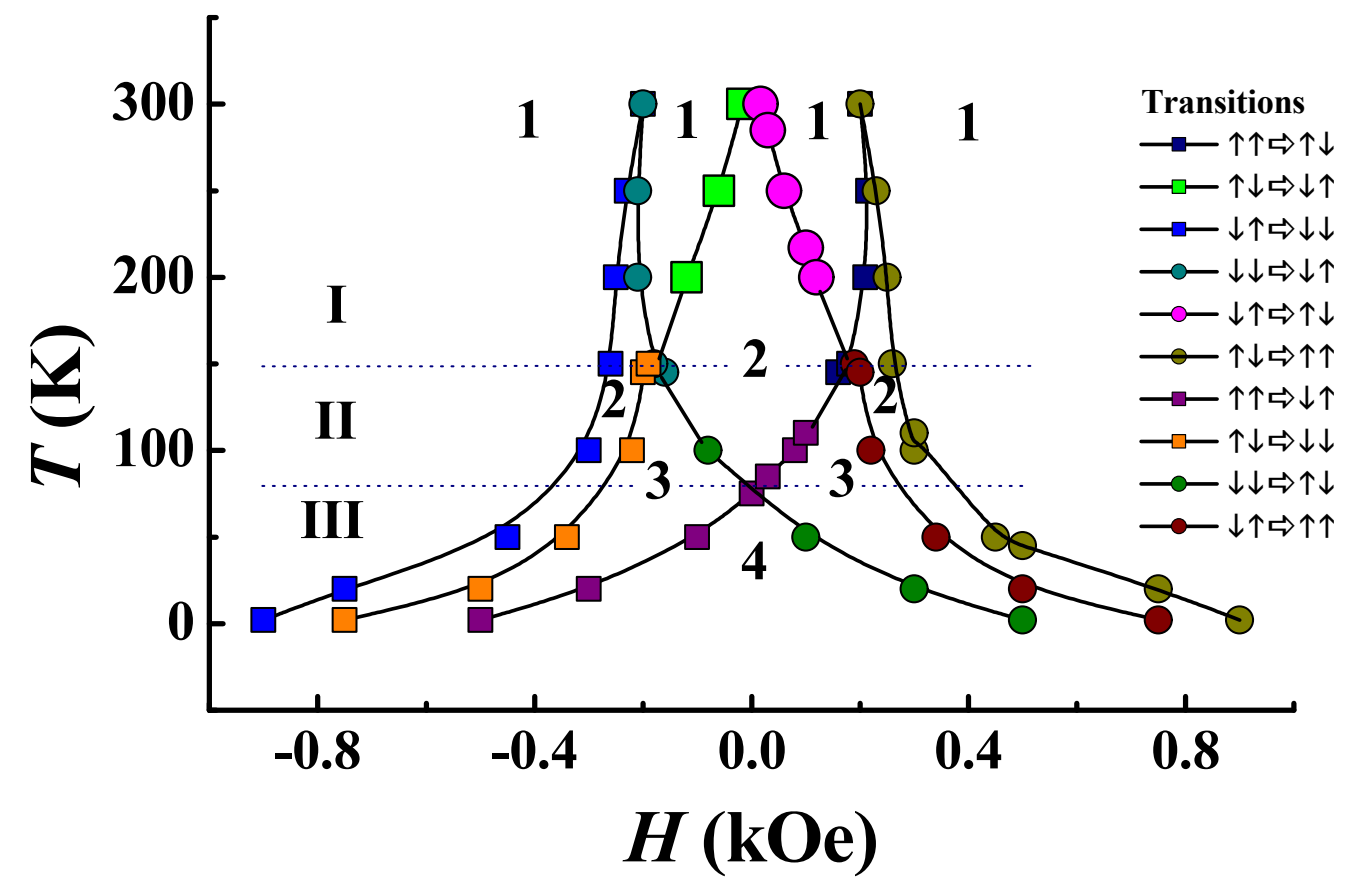

Figure 4. $H-T$ diagram of magnetization switching of $\mathrm{CoFeB} / \mathrm{Ta} / \mathrm{CoFeB}$ bilayer. Solid lines and symbols on the diagram are temperature dependences of interstate transition fields. Roman numbers correspond to the types of magnetic hysteresis loop, discussed in the text, dot lines divide temperature ranges for hysteresis loops of type I, II and III. Numeration 1-4 corresponds to number of the magnetic states, which can be stored under cooling in the determined magnetic field down to the indicated temperature. Round symbols correspond to bottom-up field sweeping direction; square symbols correspond to top-down field sweeping direction. Lines are guides for eyes.

In the following, we will explain the origin of the different loops and estimate the values of key physical interactions. In this approach we will use a macrospin approximation where the magnetization inside each layer is considered as uniform. The bilayer switching is controlled by three energies $\left.E_{\mathrm{EX}}, E_{\mathrm{eff}}, E_{\mathrm{Z}}: 1\right)$ the exchange interaction $E_{\mathrm{EX}}$ between the two ferromagnetic layers, 2) the magnetic anisotropy, which forms energy barrier $E_{\text {eff }}$ for 
magnetization reversal separating the different states, 3) the Zeeman energy $E_{Z}$ due to external applied magnetic field. Stray field contribution is negligibly small due to homogeneous magnetic field inside bilayer plane device. Just small part of the device near its edges contributes to energy balance. This part is neglected in the most literature devoted to spin valves.

Exchange energy $E_{\mathrm{EX}}$ increases with increasing of the interface area, because the interface hybridization of the atomic wave functions is origin of this energy. The sign of $E_{\mathrm{EX}}$ depends on the mutual orientation of layers' magnetic moments $\boldsymbol{M}_{\mathbf{1}}$ and $\boldsymbol{M}_{\mathbf{2}}$. We'll define $E_{\mathrm{EX}}$ $=-S J \cdot\left(\boldsymbol{M}_{\mathbf{1}} \cdot \boldsymbol{M}_{\mathbf{2}}\right) /\left|\boldsymbol{M}_{\mathbf{1}}\right| \cdot\left|\boldsymbol{M}_{\mathbf{2}}\right|$, where $J$ is exchange integral per unit of the interface area $S$. Negative value $E_{\mathrm{EX}}=S \cdot J>0$ corresponds to antiferromagnetic coupling, while $E_{\mathrm{EX}}=-S \cdot J$ $<0$ corresponds to parallel mutual orientations of bottom and top layers magnetic moments $\boldsymbol{M}_{1}$ and $\boldsymbol{M}_{2}$. The Zeeman energy of the sample is $E_{Z}=-\left(\left(\boldsymbol{m}_{\mathbf{1}} \cdot S \cdot h_{1}+\boldsymbol{m}_{\mathbf{2}} \cdot S \cdot h_{2}\right) \cdot \boldsymbol{H}\right)=\left(\boldsymbol{M}_{\mathbf{1}}+\boldsymbol{M}_{\mathbf{2}}\right) \cdot \boldsymbol{H}, \boldsymbol{m}_{\mathbf{1}}$ and $\boldsymbol{m}_{\mathbf{2}}$ are layer's magnetizations, $h_{1}$ and $h_{2}$ are bottom and top layers' widths. Energy barriers of bottom and top layer's magnetization reversals will be designated as $E_{\text {effl }}$ and $E_{\text {eff2 }}$, respectively. Exchange energy is proportional to the interface area, Zeeman energy of the each magnetic layer is proportional to the volume of this layer. All mentioned above energies are additive and unified to the same dimensionality. The total energy of bilayer is determined by the following equation:

$$
E=E_{\mathrm{EX}}+E_{\mathrm{Z}}+E_{\mathrm{eff} 1}+E_{\mathrm{eff} 2}
$$

Equation (1) applied to the defined magnetic state results in straight line in $E(H)$ coordinates. The slope of this line depends on mutual orientation of the magnetic moments $\boldsymbol{M}_{1}$ and $\boldsymbol{M}_{2}$. Different states of the bilayer has different slopes of the $E(H)$ dependences crossing in threshold points, where switching of the magnetization states occurs (Figure 5a). Positions of the crossing points and correspondent threshold magnetic fields depend on field independent terms in (1). For that reason $E_{\mathrm{EX}}, E_{\mathrm{eff} 1}$ and $E_{\mathrm{eff} 2}$ affect the threshold values shifting straight lines along energy axis. One should include into consideration all four terms in Eq. (1) to determine threshold magnetic fields. The calculated magnetic field dependences of total energy (1) for different values of the energy barrier for magnetization reversal are shown by thin solid lines in the Figure 5 a-d.

In the absence of reversal magnetization barriers the energy of the bilayer is determined by the sum of exchange and Zeeman contributions. Energy diagram for that case is shown in the Fig.5a. The current state of the bilayer is determined by the lowest energy in the sweeping magnetic field. The critical magnetic fields of transitions between the states 
correspond to the crossing points of the solid lines and can be determined by expressions:

$$
H_{\uparrow \uparrow \Longleftrightarrow_{\uparrow \downarrow}}=\left|E_{\mathrm{EX}}\right| /\left|M_{2}\right| ; H_{\uparrow \downarrow \downarrow \downarrow \uparrow}=0 ; H_{\downarrow \uparrow \uparrow \downarrow \downarrow}=-\left|E_{\mathrm{EX}}\right| /\left|M_{2}\right| .
$$

Magnetic anisotropy of the bilayer results in additional potential barrier separating magnetic states of the bilayer. Since, in our experiments, we study bilayer magnetization along easy axis, altitude of the potential barrier is not equal to anisotropy barrier. For that reason $E_{\text {eff1 }}$ and $E_{\text {eff2 }}$ in Eq. (1) do not mean magnetic anisotropy energies. In presence of the reversal magnetization barrier, transition between magnetic states occurs if difference between these states energies exceeds the barrier of the reversing layer. On the diagram, the system remains in the current state (bold solid line) until the magnetic field reaches a threshold value strong enough to overcome the energy barrier (dashed line).

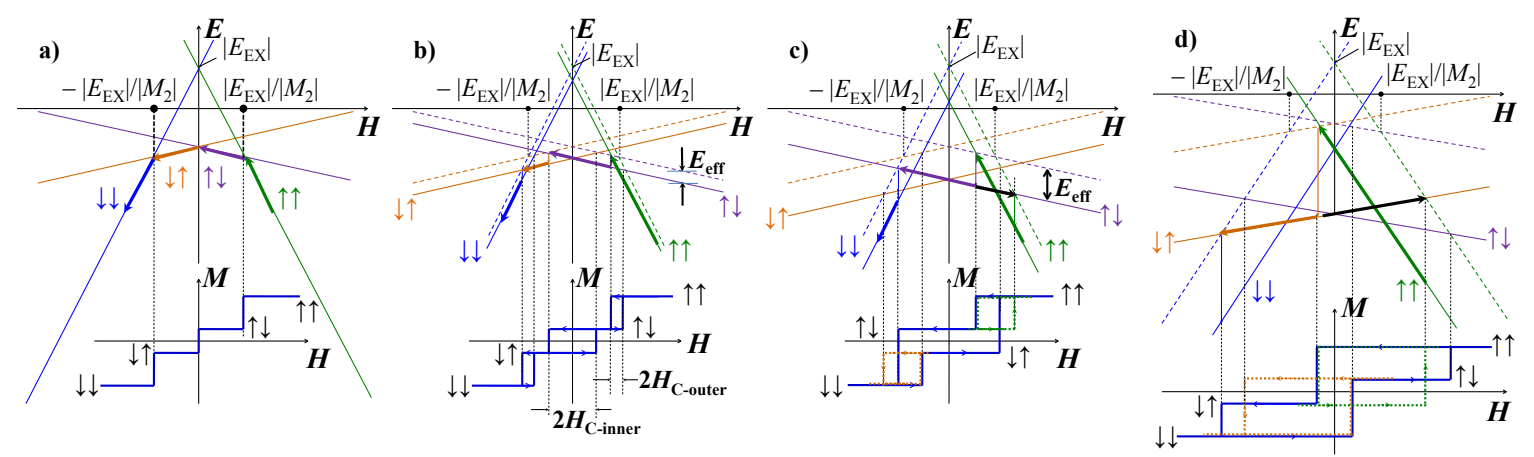

Figure 5. Energy diagrams (top panels) and simulation (bottom panels) of idealized $M(H)$ curve (zero barrier for reversal) (a), and real hysteresis loops of types I (b), II (c) and III (d) for antiferromagnetically coupled bilayer. Top panels: solid lines indicate energy curves $E(H)$ for stable magnetic states $\uparrow \uparrow$ - green, $\uparrow \downarrow-$ violet, $\downarrow \uparrow$ - orange, and $\downarrow \downarrow$ - blue curve; dashed lines are curves for the same states shifted upwards by the anisotropy barrier. The fields of transitions between the states are determined by the points where solid line for previous state crosses dashed line for the next state. Bold arrows indicate trajectory of the system for top-down field sweeping direction. Dot lines indicate the correspondence between the critical points on the energy diagrams and transition fields on the hysteresis loops. Bottom panels: blue solid line - simulated major hysteresis loop, Green and orange dashed lines in (c) and (d) - simulated minor hysteresis loops.

Threshold magnetic fields of the interstate transitions will be defined by following expressions:

$$
\begin{aligned}
& H_{\uparrow \uparrow \Rightarrow \uparrow \downarrow}=-H_{\downarrow \downarrow \Rightarrow \downarrow \uparrow}=\frac{2\left|E_{E X}\right|-E_{2 e f f}}{2 M_{2}}(3), \quad H_{\uparrow \uparrow \Rightarrow \downarrow \uparrow}=-H_{\downarrow \downarrow \Rightarrow \uparrow \downarrow}=\frac{2\left|E_{E X}\right|-E_{1 e f f}}{2 M_{1}} \\
& H_{\uparrow \downarrow \Rightarrow \downarrow \uparrow}=-H_{\downarrow \uparrow \Rightarrow \uparrow \downarrow}=\frac{E_{1 e f f}+E_{2 e f f}}{2\left(M_{2}-M_{1}\right)} \quad(5), H_{\uparrow \downarrow \Rightarrow \downarrow \downarrow}=-H_{\downarrow \uparrow \Rightarrow \uparrow \uparrow}=-\frac{2\left|E_{E X}\right|+E_{1 e f f}}{2 M_{1}} \\
& H_{\downarrow \uparrow \Rightarrow \downarrow \downarrow}=-H_{\uparrow \downarrow \Rightarrow \uparrow \uparrow}=-\frac{2\left|E_{E X}\right|+E_{1 e f f}}{2 M_{1}}(7), H_{\uparrow \uparrow \Rightarrow \downarrow \downarrow}=-H_{\downarrow \downarrow \Rightarrow \uparrow \uparrow}=\frac{E_{1 e f f}+E_{2 e f f}}{2\left(M_{2}+M_{1}\right)}(8) .
\end{aligned}
$$


Three types of the hysteresis loop are possible depending on $E_{\mathrm{EX}} / E_{1 \mathrm{eff}}, E_{\mathrm{EX}} / E_{2 \mathrm{eff}}$ and $M_{2} / M_{1}$ values.

1) Type I: $E_{1 e f f}>2\left|E_{E X}\right| \cdot \frac{M_{1}-M_{2}}{M_{2}}$.

In that case, the magnetic hysteresis loop contains three subsequent transitions $\uparrow \uparrow$ $\Rightarrow \uparrow \downarrow, \uparrow \downarrow \Rightarrow \downarrow \uparrow$ and $\downarrow \uparrow \Rightarrow \downarrow \downarrow$ for top down magnetic field sweeping direction and equivalent transitions $\downarrow \downarrow \Rightarrow \downarrow \uparrow, \downarrow \uparrow \Rightarrow \uparrow \downarrow$ and $\uparrow \downarrow \Rightarrow \uparrow \uparrow$ for bottom up magnetic field sweeping direction. Corresponding transition fields are determined from Equations (3), (5) and (7), respectively. Major hysteresis loop of the type I contains three subloops. The inner loop corresponds to simultaneous magnetization reversal of the top and bottom layers. The outer loops on the left and on the right correspond to the top layer magnetization reversal (Figs. 2a and 5b). Experimentally, the hysteresis loop of this type takes place in the $180 \mathrm{~K}-330 \mathrm{~K}$ temperature range. Coercive field of the inner loop can be expressed by Eq. (9):

$$
H_{C-\text { inner }}=\frac{E_{1 e f f}+E_{2 e f f}}{2\left(M_{1}-M_{2}\right)}
$$

Centers $H_{\mathrm{B}}$ and coercive force $H_{\mathrm{C}-\text { outer }}$ of the outer loops can be expressed by the following equations:

$$
H_{B}= \pm \frac{\left|E_{E X}\right|}{M_{2}}(10), \quad H_{C-\text { outer }}=\frac{E_{1 e f f}}{2 M_{1}}
$$

Thus, for adjusted exchange energy and barrier of reversal magnetization the coercive fields of the inner and the outer loops, as well as outer loop bias field are determined by the ratio of magnetic moments of the top and the bottom layers. Expressions (9), (10) allow us to conclude that central (inner) subloop is always wider in comparison with side (outer) subloops, because the ratio of the coercive fields of the central and side subloops is controlled by the ratio of the magnetic moments and correspondent thicknesses of the layers. Magnetic moment of the thin layer $M_{1}$ controls bias field, while magnetic moment of the thick layer $M_{2}$ controls coercive fields of the outer loops, difference of the $M_{1}$ and $M_{2}$ values determines coercive field of the inner loop. Close values of the $M_{1}$ and $M_{2}$ result in difference $M_{1}-M_{2}$ smaller than $M_{1}$ and $M_{2}$, both. 
For that reason coercive field of the inner loop can be different from coercive fields of the outer loops up to several times even at the same reversal magnetization barriers of the layers. This case takes place in our experiments (Figs.2a, 5b and 6).

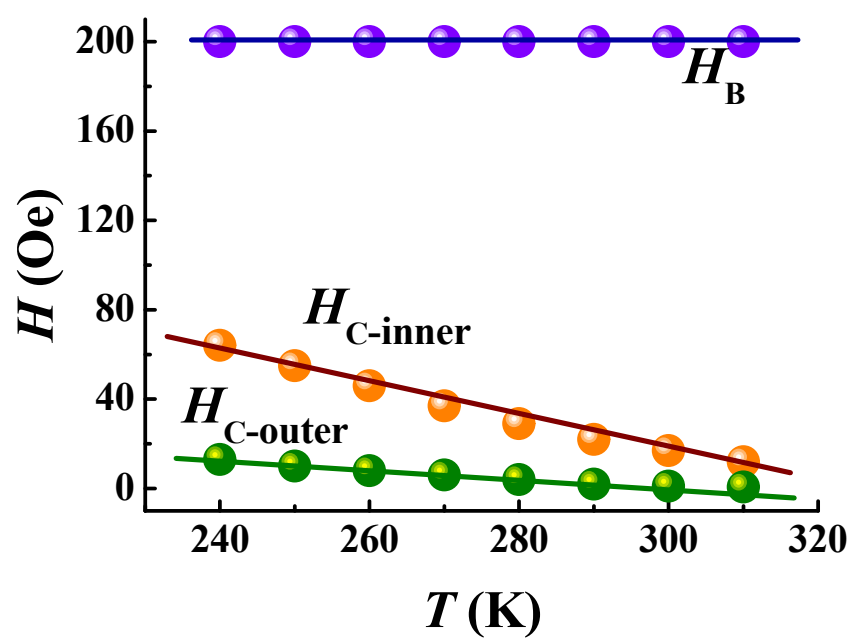

Figure 6 . Temperature dependences of hysteresis parameters: coercive fields $H_{\mathrm{C} \text {-inner }}, H_{\mathrm{C}-\text { outer }}$ and bias field $H_{\mathrm{B}}$. Solid lines are linear approximations.

Equations (9) allow us to estimate the exchange energy and magnetization reversal barriers at $T=300 \mathrm{~K}: E_{\mathrm{EX}} / S=-0.01 \mathrm{erg} / \mathrm{cm}^{2}(S-$ the interface area $), E_{\text {leff }} / S=4.0 \cdot 10^{-3}$ $\mathrm{erg} / \mathrm{cm}^{2}, E_{2 \mathrm{eff}} / S=2.5 \cdot 10^{-3} \mathrm{erg} / \mathrm{cm}^{2}$. The interlayer exchange coupling constant $E_{\mathrm{EX}} / S$ has been obtained for the opposite directions of the layers magnetizations by evaluating the magnetic field in the center of the two outer hysteresis loops. Estimated barrier heights $E_{1 \text { eff }} / S$ and $E_{2 \text { eff }} / S$ are very close to the interface anisotropy constants, determined in [36] for hard axis magnetization measurements in the identical sample. This allows one to conclude that the dominant contribution to the magnetization reversal barrier results from interface anisotropy.

2) Type II: $E_{2 e f f} \cdot \frac{M_{1}}{M_{2}}-2\left|E_{E X}\right| \cdot \frac{M_{1}-M_{2}}{M_{2}}<E_{1 e f f} \leq 2\left|E_{E X}\right| \cdot \frac{M_{1}-M_{2}}{M_{2}}$

Magnetic hysteresis loop contains two transitions instead of three ones (Fig. 5c). These transitions correspond to remagnetization of the top layer from parallel state in magnetic field $H_{\uparrow \uparrow} \Rightarrow \uparrow \downarrow(3)$ and remagnetization of the bottom layer from antiparallel state in magnetic field $H_{\uparrow \downarrow} \Rightarrow \downarrow \downarrow$ (6). In the studied bilayer this mode of hysteresis loop takes place in the $120 \mathrm{~K}-170 \mathrm{~K}$ temperature range (Fig. $2 \mathrm{~b}$ and Table 1). 
3) Type III: $E_{1 e f f}<E_{2 e f f} \cdot \frac{M_{1}}{M_{2}}-2\left|E_{E X}\right| \cdot \frac{M_{1}-M_{2}}{M_{2}}$

Similarly with previous case, the hysteresis loop of type III contains two transitions, but the order of layer switching is opposite with order of switching of type II. First the bottom layer (with larger magnetic moment) is switched and then the upper layer is switched in higher magnetic field (Figures $2 \mathrm{c}$ and $5 \mathrm{~d}$ ). Magnetic fields of the switching can be expressed by Equations (4) and (7). This mode of hysteresis loop takes place in the $2 \mathrm{~K}-110 \mathrm{~K}$ temperature range (Fig. 2c and Table 1).

Proposed diagram technique allows simulating minor hysteresis loops. For the loop of the type II (Fig. 5c) change of the direction of magnetic field sweep from top-down to bottom-up in the point $\mathbf{A}$ on the $E(H)$ curve (black arrow on the violet line) will not change $\uparrow \downarrow$ state until the barrier is not overcome. Transition from $\uparrow \downarrow$ to $\uparrow \uparrow$ will occur, when violet solid line (corresponding to $\uparrow \downarrow$ state) crosses green dashed line. Simulated minor hysteresis loops correspondent to this transition (and equivalent one in negative magnetic fields), are given at the bottom panel in the Fig. 5c. Analogous simulation is possible for the minor hysteresis loops of type III (black arrow on the diagram and green minor loops at the bottom panel in the Fig.5d).

Proposed method allows us to obtain two additional loop configurations of the type III. If simultaneously two conditions are fulfilled ( $E_{2 \mathrm{eff}}>E_{1 \mathrm{eff}}, 2\left|E_{\mathrm{EX}}\right|>E_{1 \mathrm{eff}}$ ), the transition from $\uparrow \uparrow$ to $\downarrow \uparrow$ occurs in positive magnetic field resulting in the "butterfly" shape of the hysteresis loop of the type III (Figure S3 a). In our experiments, such hysteresis loop was observed at $100 \mathrm{~K}$ (Figure S3 c). Another loop configuration is possible if $H_{\uparrow \uparrow} \Rightarrow$ $\downarrow \uparrow$ and $H_{\uparrow \uparrow} \Rightarrow \uparrow \downarrow$ threshold fields are equal. In the diagram this case corresponds to the intersection of the $\uparrow \uparrow$ state (green solid line) with $\uparrow \downarrow$ and $\downarrow \uparrow$ states shifted along energy axis to reversal barrier energy of the top and bottom layer, respectively (violet and orange dashed lines), at the same point. This 'triple point' corresponds to the bifurcation between loops of the type II and type III resulting in loop containing two transitions, which intermediate state is between $\uparrow \downarrow$ and $\downarrow \uparrow$ states. Diagram and simulation for that case are given in the supporting materials (Figure S3 b). This type of hysteresis loop is observed at 115 K (Figure S3 d). 
Thus, proposed diagram allows us to describe all configurations of the hysteresis loops and transitions between magnetic states in the entire $2-300 \mathrm{~K}$ temperature range. This technique allows to establish, is transition permitted or forbidden and to determine threshold values of the magnetic fields for permitted transitions. Finally, the relations between magnetization reversal barriers, transition fields and layers' magnetic moment can be used to estimate exchange energy and magnetization reversal energy barriers from experimental $M(H)$ curves, recorded in easy-axis (out-of-plane) orientation.

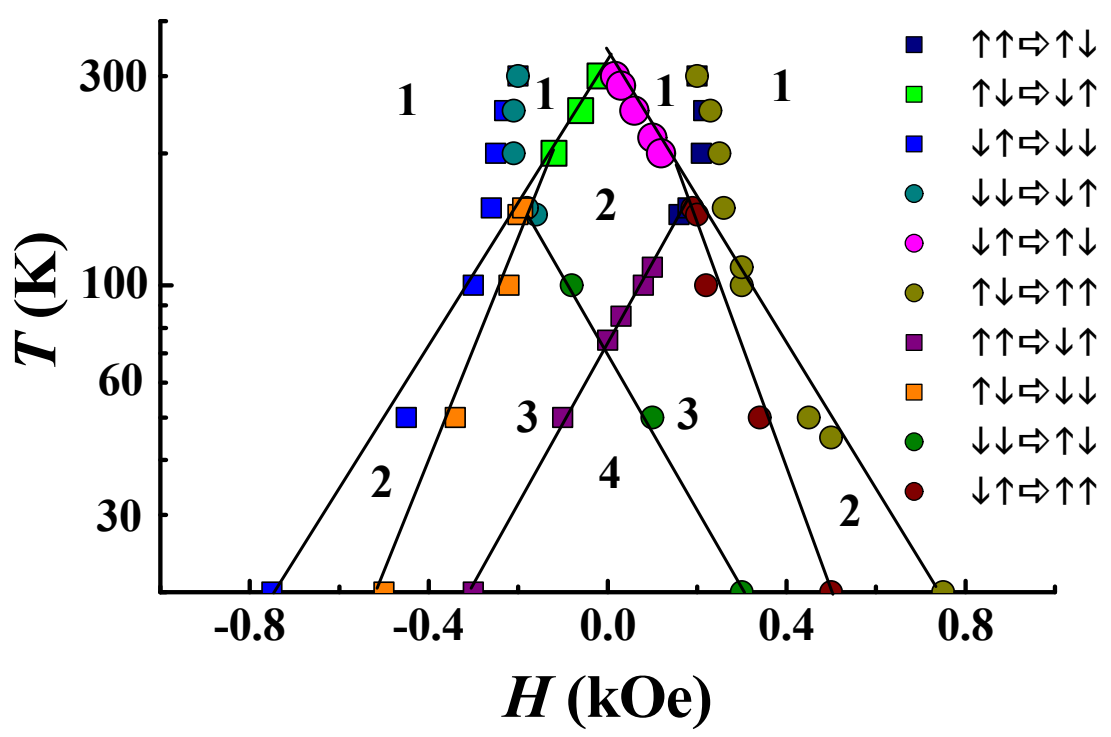

Figure 7. $H-T$ diagram of different magnetization states existence in the $\mathrm{CoFeB} / \mathrm{Ta} / \mathrm{CoFeB}$ bilayer in $H-T$ space in $\log (T)$ scale. Solid lines are linear approximations.

Cross-border lines on the map of the magnetic states (Figure 4) can be more or less straightened if the map is presented in semi logarithmic coordinates (Figure 7). In the frames of the considered model combining macrospin and intermediate domain wall states, one can estimate temperature dependences of magnetization reversal barrier of top and bottom layers magnetization reversal. (Figure S4 in supplementary material). These parameters manifest exponential decrease under heating.

\section{CONCLUSIONS}

Magnetization states and transitions for perpendicularly magnetized antiferromagnetically exchange coupled $\mathrm{CoFeB} / \mathrm{Ta} / \mathrm{CoFeB}$ bilayers have been studied. Those states and transitions are summarized in a $H-T$ magnetization switching diagram. The sets of permitted and forbidden interstate magnetization transitions are different for $2 \mathrm{~K}-110 \mathrm{~K}$, 
$120 \mathrm{~K}-170 \mathrm{~K}$ and $180 \mathrm{~K}-300 \mathrm{~K}$ temperature ranges. The threshold magnetic fields of magnetization switching and the correspondent magnetic hysteresis loop are explained by the competition between interlayer exchange coupling, Zeeman energy and energy barrier of layer's magnetization reversal. Exchange and magnetization reversal barrier parameters were extracted by the of temperature and field dependences of inter-state transition fields.

\section{SUPPLEMENTARY MATERIAL}

See supplementary material for temperature dependences of magnetic moment under the sample heating (Fig. S1) and cooling (Fig. S2) in different magnetic fields, simulation and experiment for butterfly shape of magnetic hysteresis loop of the type III (Fig. S3) and temperature dependences of the top and bottom layers magnetization reversal barriers (Fig. S4).

\section{ACKNOWLEDGMENTS}

The work was supported by the joint French National Research Agency (ANR)-National Natural Science Foundation of China (NSFC) SISTER project (Grants No. ANR-11-IS100001 and No. NNSFC 61161130527) and ENSEMBLE project (Grants No. ANR-14-0028-01 and No. NNSFC 61411136001) as well as by Région Lorraine. And by the ANR-NSF Project, ANR-13-IS04-0008-01, COMAG by the ANR-Labcom Project LSTNM and by the Université de la Grande Region (UniGR funded P. Pirro Post-Doc). Experiments were performed using equipment from the TUBE - Davm funded by FEDER (EU), ANR, the Region Lorraine, and Grand Nancy.A.T and R.M. were supported by Ministry of Education and Science of Russian Federation, grant No. 3.1992.2017/ПЧ and agreement No. 14.W03.31.0001.

\section{REFERENCES}


${ }^{1}$ L. Cuchet, B. Rodmacq, S.Auffret, R.C. Sousa, I.L. Prejbeanu and B. Dieny, $\underline{\text { Sci. Rep. }}$ 6, 21246 (2016).

${ }^{2}$ M. Kuteifan, M. Lubarda, S. Fu, R. Chang, M.A. Escobar, S. Mangin, E. Fullerton, and V. Lomakin, AIP Advances 6, 045103 (2016).

${ }^{3}$ S. Alebrand, M. Gottwald, M. Hehn, D. Steil, M. Cinchetti, D. Lacour, E.E. Fullerton, M. Aeschlimann, and S. Mangin, Appl. Phys. Lett. 101, 162408 (2012).

${ }_{4}^{4}$ S. Mangin, M. Gottwald, C-H. Lambert, D. Steil, L. Pang, M. Hehn, S. Alebrand, M. Cinchetti, G. Malinowski, Y. Fainman, M. Aeschlimann, and E.E. Fullerton, Nature Mat. 13, 286 (2014).

${ }^{5}$ G. A. Wang, S. Nakashima, S. Arai, T. Kato, and S. Iwata. J. Appl. Phys. 107, 09E709 (2010).

${ }^{6}$ S. Ikeda, K. Miura, H. Yamamoto, K. Mizunuma, H. D. Gan, M. Endo, S. Kanai, J. Hayakawa, F. Matsukura and H.Ohno. Nat. Mat. 9, 721 (2010).

${ }^{7}$ X. Wang. Metallic Spintronic Devices. CRC Press, (2014).

${ }^{8}$ R. Valenzuela, J.J. Freijo, A. Salcedo, M. Vázquez and A. Hernando, J. Appl. Phys. 81 4301 (1997).

${ }^{9}$ S. A. Manuilov, A. M. Grishin, and M. Munakata. J. Appl. Phys. 109, 083926 (2011).

${ }^{10}$ J.M. Teixeira, R.F.A. Silva, J. Ventura, A.M. Pereira, F. Carpinteiro, J.P. Ara'ujo, J.B. Sousa, S. Cardoso, R. Ferreira, P.P. Freitas. Mat. Sci. and Eng. B 126, 180 (2006).

${ }^{11}$ J.-P. Tetienne, T. Hingant, J.-V. Kim, L. Herrera Diez, J.-P. Adam, K. Garcia, J.-F. Roch, S. Rohart, A. Thiaville, D. Ravelosona, V. Jacques. Science 344, 1366 (2014).

${ }^{12}$ S. Peng, M. Wang, H. Yang, L. Zeng, J. Nan, J. Zhou, Y. Zhang, A. Hallal, M. Chshiev, K.L. Wang, Q. Zhang, W. Zhao. Sci. Rep. 5, 18173 (2015).

${ }^{13}$ L. Heyne, M. Kläui, D. Backes, P. Möhrke, T. A. Moore, J. G. Kimling, O. Boulle, U. Rüdiger, L. J. Heyderman, A. Fraile Rodríguez, F. Nolting, K. Kirsch, and R. Mattheis. J. Appl. Phys. 103, 07D928 (2008).

${ }^{14}$ Jaehun Cho, Jinyong Jung, Shin-Yong Cho, Chun-Yeol You. Effect of annealing temperature on exchange stiffness of CoFeB thin films. J. Magn. Magn. Mat. 395, 18 (2015). ${ }^{15}$ S. Fukami, T. Suzuki, Y. Nakatani, N. Ishiwata, M. Yamanouchi, S. Ikeda, N. Kasai, and H. Ohno. Appl. Phys. Lett. 98, 082504 (2011).

${ }^{16}$ C. J. Durrant, R. J. Hicken, Q. Hao, and G. Xiao. Phys. Rev. B 93, 014414 (2016)

${ }^{17}$ J. Wells, J.H.Lee, R.Mansell, R.P.Cowburn, O.Kazakova. J. Magn. Magn. Mat. 400, 219 (2016). 
18 E. Albisetti, D. Petti, J. Magn. Magn. Mat. 400, 230 (2016).

${ }^{19}$ L. Herrera Diez, F. García-Sánchez, J.-P. Adam, T. Devolder, S. Eimer, M. S. El Hadri, A. Lamperti, R. Mantovan, B. Ocker, and D. Ravelosona. Appl. Phys. Lett. 107, 032401 (2015).

${ }^{20}$ L. You, O. Lee, D. Bhowmik, D. Labanowski, J. Hong, J. Bokor, and S. Salahuddin, PNAS 112, 10310 (2015).

${ }^{21}$ H. Ohno, D. Chiba, F. Matsukura, T. Omiya, E. Abe, T. Dietl, Y. Ohno, K. Ohtani. Nature 408, 944 (2000).

${ }^{22}$ D. Chiba, M. Sawicki, Y. Nishitani, Y. Nakatani, F. Matsukura and H. Ohno. Nature 455, 515 (2008).

${ }^{23}$ E. B. Myers, D. C. Ralph, J. A. Katine, R. N. Louie, R. A. Buhrman, Science 285, 867 (1999).

${ }^{24}$ D. C. Ralph and M. D. Stiles, J. Magn. Magn. Mater. 320, 1190 (2008).

${ }^{25}$ A. Chernyshov, M. Overby, X. Liu, J. K. Furdyna, Y. Lyanda-Geller and L. P. Rokhinson, Nature Phys. 5, 656 (2009).

${ }^{26}$ H. Sato, M. Yamanouchi, S. Ikeda, S. Fukami, F. Matsukura, and H. Ohno. Appl. Phys. Lett. 101, 022414 (2012).

${ }^{27}$ C.C. Tsai, Chih-Wei Cheng, Meng-Chiau Tsai, and G. Chern. IEEE Trans. Magn. 50, 1401404 (2014).

${ }^{28}$ T. Zhu, P. Chen, Q. H. Zhang, R. C. Yu, and B. G. Liu. Appl. Phys. Lett. 104, 202404 (2014).

${ }^{29}$ X. Qiao, B. Wang, Z. Tang, Y. Shen, H. Yang, J. Wang, Q. Zhan, S. Mao, X. Xu, and R.W. Li, AIP Advances 6, 056106 (2016).

${ }^{30}$ W. X. Wang, Y. Yang, H. Naganuma, Y. Ando, R. C. Yu, and X. F. Han, Appl. Phys. Lett. 99, 012502 (2011).

${ }^{31}$ R. Soucaille, M. Belmeguenai, J. Torrejon, J.-V. Kim, T. Devolder, Y. Roussign'e, S.-M. Ch'erif, A. A. Stashkevich, M. Hayashi, and J.-P. Adam, Phys. Rev. B 94, 104431 (2016). 32 J. Torrejon, J. Kim, J. Sinha, S. Mitani, M. Hayashi, M. Yamanouchi and H. Ohno. Nat. Comm. 5, 4655 (2014).

${ }^{33}$ N. Lei, T. Devolder, G. Agnus, P. Aubert, L. Daniel, J.V. Kim, W. Zhao, T. Trypiniotis, R. P. Cowburn, C. Chappert, D. Ravelosona, P. Lecoeur, Nat. Comm. 4, 1378 (2013).

${ }^{34}$ G. Yu, Z. Wang, M. Abolfath-Beygi, C. He, X. Li, K. L. Wong, P. Nordeen, H. Wu, G. P. Carman, X. Han, I. A. Alhomoudi, P. K. Amiri, and K. L. Wang, Appl. Phys. Lett. 106, 072402 (2015). 
${ }^{35}$ R. Lavrijsen, A. Fernandez-Pacheco, D. Petit, R. Mansell, J. H. Lee, and R. P. Cowburn. Appl. Phys. Lett. 100, 052411 (2012).

${ }^{36}$ P. Pirro, A. Hamadeh, M. Lavanant-Jambert, T. Meyer, B. Tao, E. Rosario, Y. Lu, M. Hehn, S.Mangin, and S. Petit Watelot. arXiv:1610.02550v1 [cond-mat.mtrl-sci].

${ }^{37}$ F. Canet, S. Mangin, C. Bellouard, M. Piecuch. Euro. phys. Lett. 52, 594 (2000).

${ }^{38}$ S.M. Watson, T. Hauet, J.A. Borchers, S. Mangin, E.E. Fullerton. Appl. Phys. Lett. 92, 202507 (2008).

${ }^{39}$ S. Mangin, L. Thomas, F. Montaigne, W. Lin, T. Hauet, Y. Henry. Phys. Rev. B. 80, 224424 (2009).

${ }^{40}$ B. S. Tao, P. Barate, J. Frougier, P. Renucci, B. Xu, A. Dje_al, H. Jaffès, J.-M. George, X. Marie, S. Petit-Watelot, S. Mangin, X. F. Han, Z. G. Wang, and Y. Lu, Appl. Phys. Lett. 108, 152404 (2016).

${ }^{41}$ S.H. Liang, T.T. Zhang, P. Barate, J. Frougier, M. Vidal, P. Renucci, B. Xu, H. Jaffrès, J.M. George, X. Devaux, M. Hehn, X. Marie, S. Mangin, H.X. Yang, A. Hallal, M. Chshiev, T. Amand, H.F. Liu, D.P. Liu, X.F. Han, Z.G. Wang, and Y. Lu, Phys. Rev. B 90, 085310 (2014).

${ }^{42}$ D.B. Gopman, D. Bedau, S. Mangin, E. E. Fullerton, J. A. Katine, and A. D. Kent. J. Appl. Phys. 115, 113910 (2014).

${ }^{43}$ S. Mangin, A. Sulpice, G. Marchal, C. Bellouard, W. Wernsdorfer, and B. Barbara. Phys. Rev. B 60, 1204 (1999).

${ }^{44}$ H. Yang, M. Chshiev, B. Dieny, J. Lee, A. Manchon, and K. Shin, Phys. Rev. B 84, 054401 (2011).

${ }^{45}$ C.-W. Cheng, C.H. Shiue, T.-I. Cheng, and G. Chern, J. Appl. Phys. 112, 033917 (2012). 
MgO

CoFeB $0.8 \mathrm{~nm}^{-\mathrm{M}_{2}}$

Ta $0.75 \mathrm{~nm}$

CoFeB $1.1 \mathrm{~nm} \quad M_{1}$

MgO

GaAs 

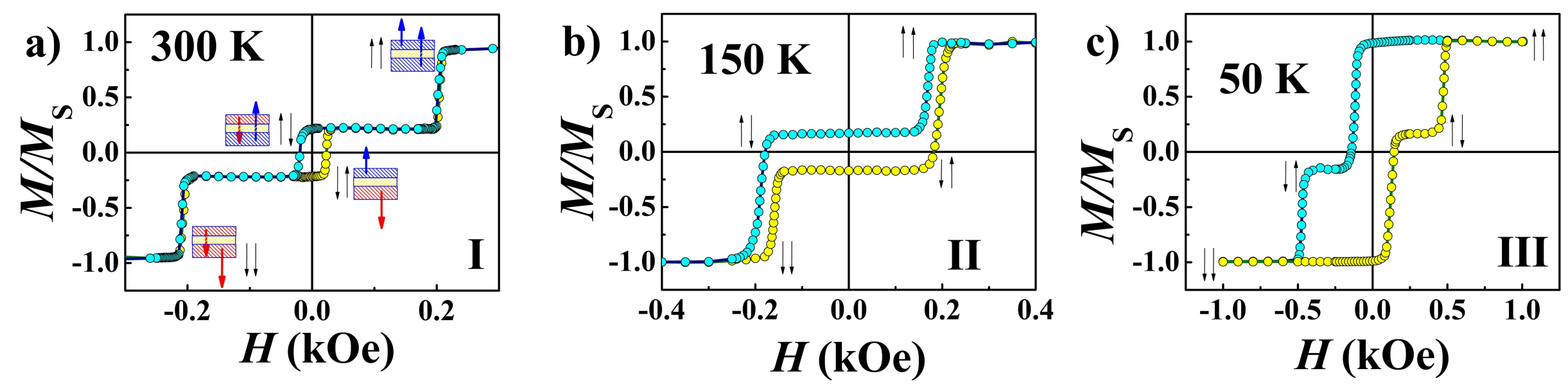

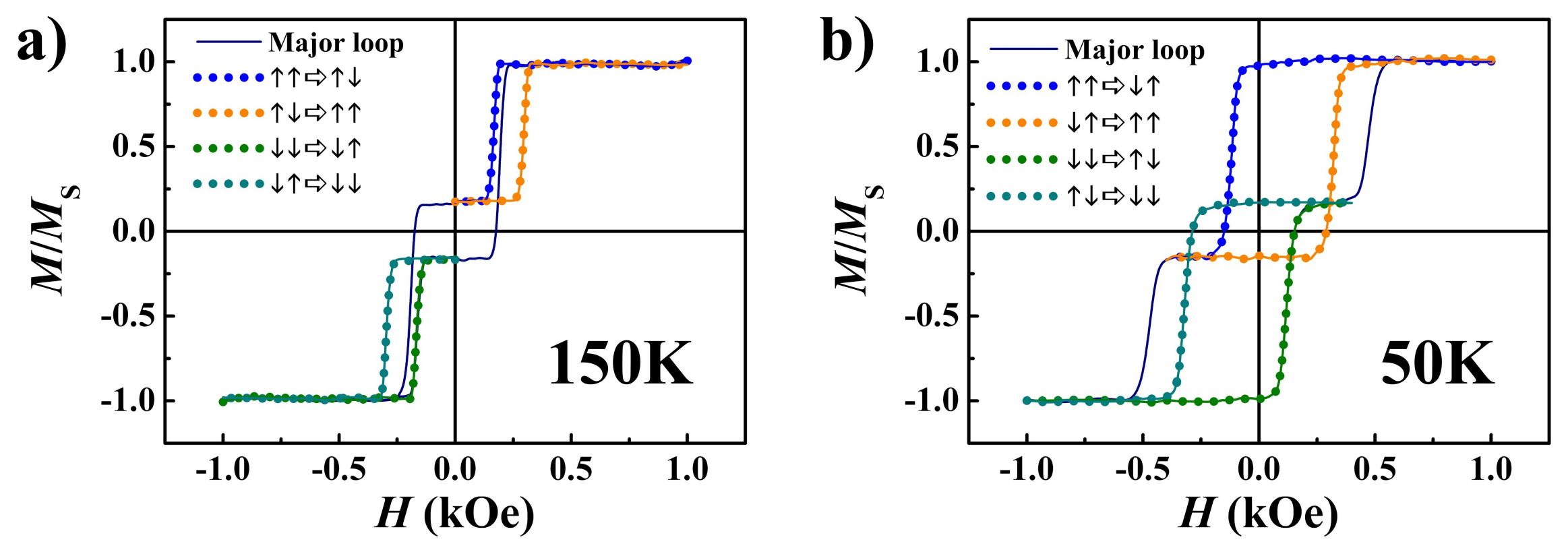


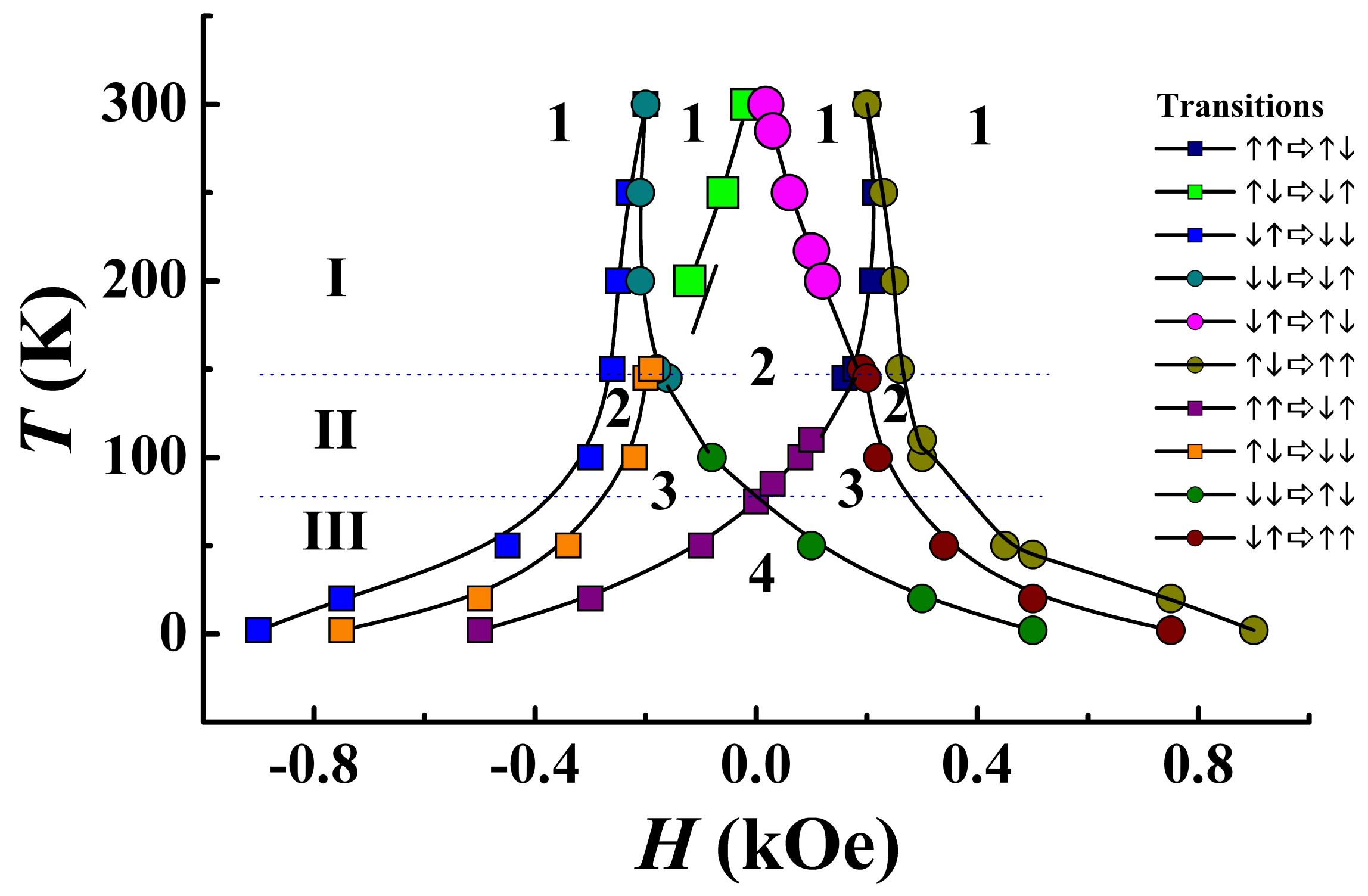



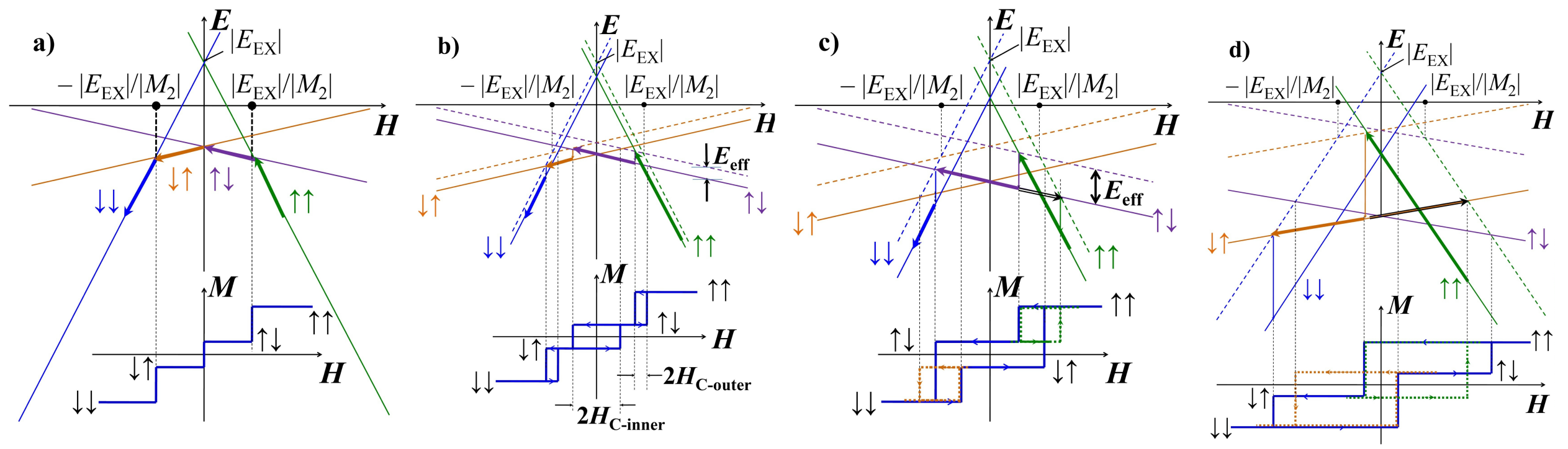


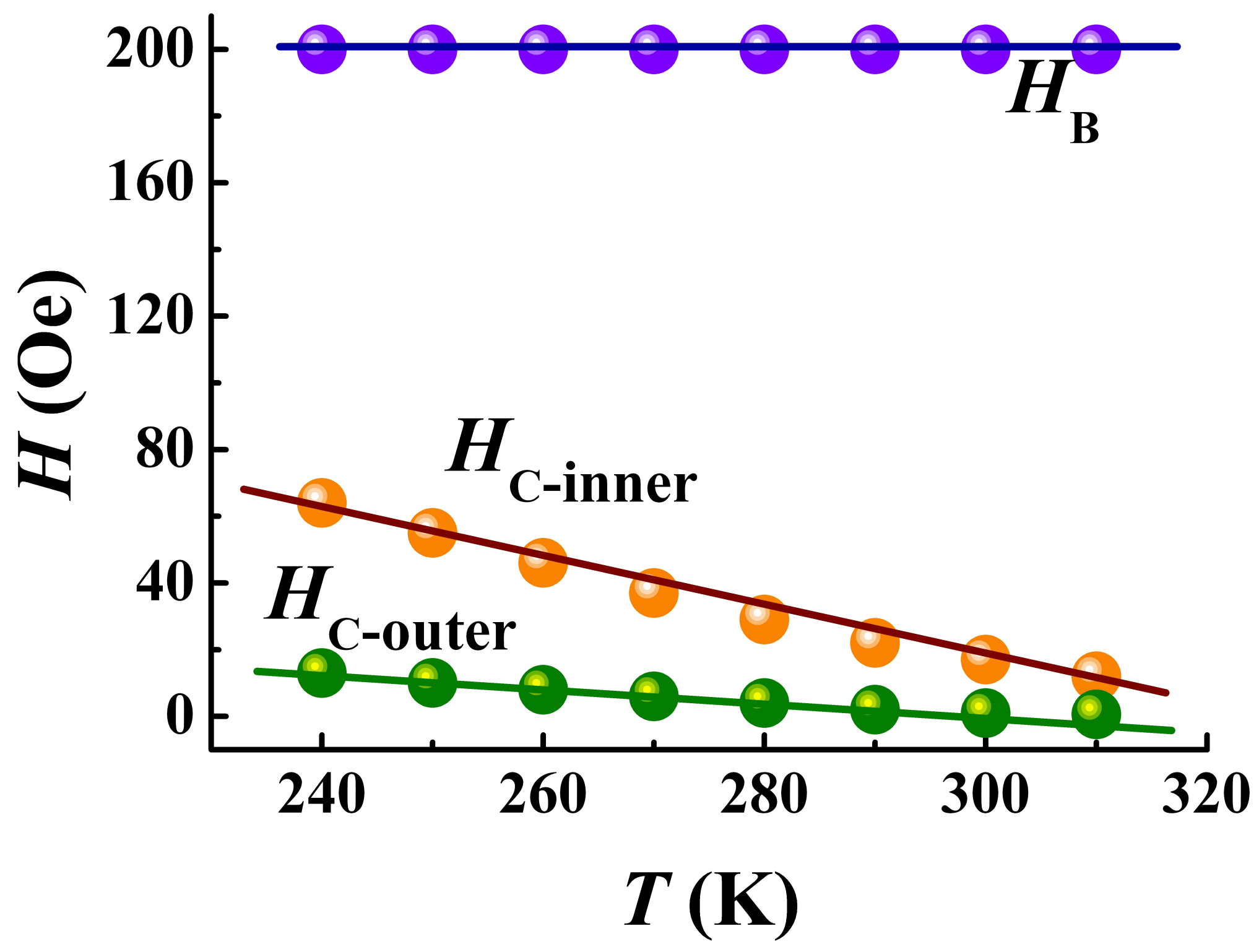




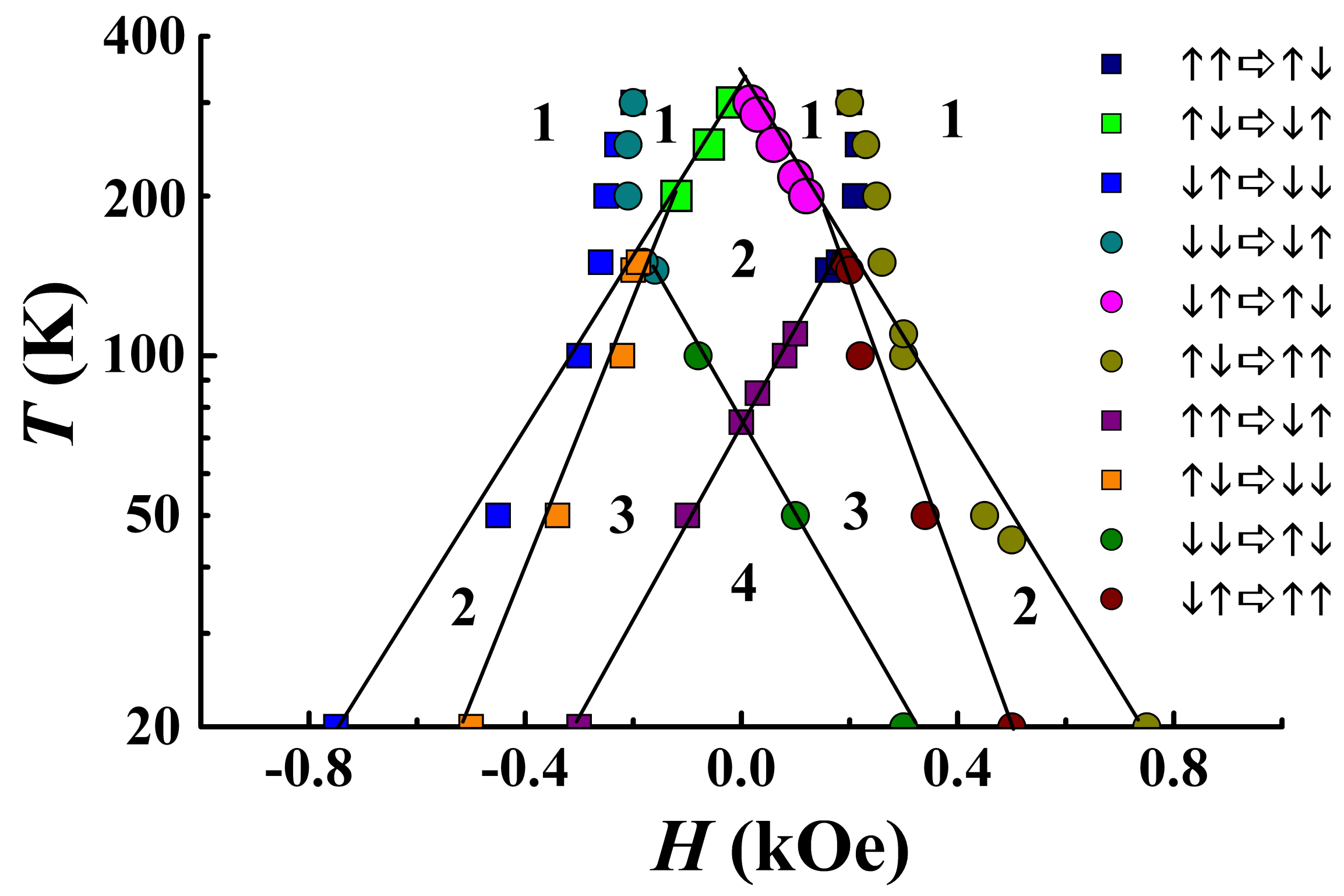


1) Anisotropy, Zeeman and exchange energy determine sequence of magnetic transitions.

2) Three temperature ranges manifest different shapes of the hysteresis loop.

3) The critical transition fields are temperature dependent. 